\title{
Current status and research progress of minimally invasive surgery for flail chest (Review)
}

\author{
HONGGANG XIA ${ }^{1,2^{*}}$, DEQING ZHU ${ }^{1 *}$, JING LI ${ }^{3}$, ZHONGYI SUN ${ }^{1}$, LIMIN DENG ${ }^{1}$, PENGZHI ZHU $^{1}$,

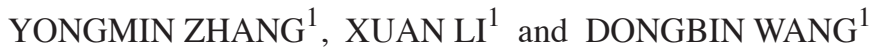 \\ ${ }^{1}$ Department of Cardiothoracic Surgery, Tianjin Hospital Affiliated to Tianjin University; \\ ${ }^{2}$ School of Medical Engineering and Translational Medicine, Tianjin University; \\ ${ }^{3}$ Teaching and Research Division, Tianjin Medical College, Tianjin 300000, P.R. China
}

Received May 17, 2019; Accepted September 25, 2019

DOI: $10.3892 /$ etm.2019.8264

\begin{abstract}
Chest trauma accounts for $\sim 13.5 \%$ of all traumas, and direct death from chest trauma accounts for $20-25 \%$ of all traumatic deaths. Chest trauma is the second cause of death from trauma. Frequent rib fractures, especially in patients with flail chest, often cause severe pain, chest wall softening, abnormal breathing and severe lung contusion and laceration, usually requiring thoracic surgery. In recent years, the open reduction and internal fixation treatment of rib fractures with flail chest has achieved satisfactory results, and some surgical indications have reached consensus. A number of scholars and medical centers have demonstrated the practicality and cost-effectiveness of rib fixation in flail chest, including the small incidence of pulmonary complications, the short ICU mechanical ventilation time, and the reduction of digestive tract inhibition. Open reduction and internal fixation of rib fractures involves multiple ribs. Conventional rib fractures require a large incision to achieve satisfactory exposure. Chest wall muscles, blood vessels and nerves (long thoracic and thoracodorsal nerves) are injured, resulting in a high infection rate of the incision and postoperative dysfunctions, such as limited upper limb, shoulder and back function, and long time numbness on the affected side of the chest. Therefore, the damage of muscles and nerves caused by conventional surgical methods limits the development of such surgical technique. Although the video-assisted thoracoscopic technique has become a necessary technical means for the treatment of thoracic trauma and has been applied to thoracic exploration and hemostasis, there is no report on the application of open
\end{abstract}

Correspondence to: Dr Dongbin Wang, Department of Cardiothoracic Surgery, Tianjin Hospital Affiliated to Tianjin University, 406 Jiefang South Road, Hexi, Tianjin 300000, P.R. China E-mail:wf8i16@163.com; 13516263681@163.com

*Contributed equally

Key words: flail chest, minimally invasive technique, open reduction and internal fixation reduction and internal fixation for rib fracture. The difficulty lies in the tightly combined bony thorax and the soft tissue of the chest wall. Therefore, experts have explored a variety of minimally invasive surgical methods for the flail chest. The current status and research progress of minimally invasive surgery for thoracic surgery are reviewed.

\section{Contents}

1. Introduction

2. Small incision rib fracture open reduction and internal fixation

3. Tunnel-type titanium alloy rib locking plate rib fracture internal fixation

4. Video-assisted thoracoscopic assisted minimally invasive rib fracture open reduction and internal fixation

5. The Su total thoracoscopic rib fracture bone plate nail intrathoracic implantation and fixation

6. Minimally invasive Nuss surgery for patients with severe flail chest with sternal fracture

7. Introduction of internal fixation materials suitable for different surgical methods

8. Conclusion

\section{Introduction}

Direct death from chest trauma accounts for $20-25 \%$ of all traumatic deaths, and chest trauma is the second leading cause of death from trauma (1). Multiple rib fracture patients, especially with flail chest, often suffer severe pain, chest wall softening, abnormal breathing and severe lung contusion and laceration, that may lead to respiratory failure, which is life threatening and often requires thoracic surgery (2). In recent years, the treatment of rib fractures with open reduction and internal fixation has achieved satisfactory results, and some surgical indications have reached consensus in the field (3-8); a number of scholars and medical centers have carried out retrospective studies, which have demonstrated the practicality and cost-effectiveness of rib fixation in patients with flail chest, including the small incidence of pulmonary complications, 
the short ICU mechanical ventilation time, the reduction in bed rest and use of large doses of analgesics and antibiotics, the indirect reduction of digestive tract inhibition, the low mortality rate and low hospitalization costs. Conventional rib fracture open reduction and internal fixation surgery causes great trauma, therefore, in recent years, experts have explored a variety of minimally invasive surgeries for flail chest (9).

\section{Small incision rib fracture open reduction and internal fixation}

Since thoracic surgery often involves multiple ribs, the conventional rib fracture open reduction and internal fixation requires a large incision to obtain satisfactory exposure. Part of the chest wall muscles, blood vessels and nerves (long thoracic and thoracodorsal nerves) are usually damaged during the procedure, resulting in high infection rate of the incision and postoperative dysfunction, such as limited upper limb, shoulder and back function, and numbness of the affected side of the chest for a long time. Therefore, the damage of conventional surgical methods on the muscles and nerves limit the development of such surgical technique. Consequently, the rational choice of the incision plays an important role in the rehabilitation process of the patients. Chinese scholars have divided the different parts of the rib fracture into subdivisions based on the anatomical features of the thorax.

Taylor et al (10) summarized the surgical approaches as: i) Standard posterolateral thoracotomy: Through this approach the posterior, lateral, and posterolateral sides of the rib fracture ends are accessible. Standard posterolateral thoracotomy can also be extended laterally or diagonally forward. The incision is curved, often reflecting the anterior ribs, and the fracture of the anterior rib can also be exposed by a percutaneous-assisted small incision. ii) 'Auscultation triangle' incision: The retractor is used to retract the muscle tissue and the scapula to minimize the damage caused by the operation; the deep muscle layer is sutured and the drainage placed to prevent 'dead cavity' and postoperative hematoma formation. iii) Trans-axillary incision: It is more suitable for anterior lateral thoracic wall rib fixation. Some muscle tissues may be disconnected as needed, the long thoracic nerve should be protected. iv) Under the breast or the pectoral muscles: The incision is along the lower edge of the breast. The breast tissue and the pectoral muscles are pulled up, and the ribs and costal cartilage of the anterior chest wall are exposed. The incision can be extended to the posterior lateral of the axillary, or extended to the standard posterolateral thoracic incision. According to the local anatomical features of different regions and rib fractures, the incision is selected and designed, the anatomical muscle space and muscle fibers are freed, and the peripheral nerves, blood vessels, surgical sites, surgical incisions and corresponding blood vessels, muscles and nerves are protected (Table I).

The specific surgical procedures are as follows: The patient is treated with general anesthesia. The specific position of the operation and the position of the incision are determined by the location of the fracture. The skin and subcutaneous tissue are removed layer by layer, and the tissue is completely freed. The peripheral blood vessels and nerves are protected. The protection of the long thoracic nerve, thoracodorsal, intercostobrachial and intercostal nerve cutaneous branches and thoracic and thoracodorsal vessels is required (Fig. 1). The intermuscular space or muscle fibers are followed to the deep part, exposing the ribs and fracture ends. The exfoliation of the intercostal muscle is performed on the upper edge of the rib. When the exfoliation is performed, the periosteum is retained as much as possible to promote the healing of the fracture. The thoracic cavity can be entered through the exfoliation point for internal fixation surgery, thoracic cavity exploration, and hemothorax removal. If fixed with a rib-embracing device, the intercostal nerve and vessels are freed, the fracture ends are repositioned, and the fixation is placed. If the locking plate is used for fixation, the fracture end can be directly restored, and the locking plate can be directly placed. Two screws are placed and locked at the two ends of the fracture, and the titanium alloy rib locking plate is firmly fixed. Satisfactory surgical results can be obtained. Exposure limits are often applied to $<5$ consecutive rib fractures.

\section{Tunnel-type titanium alloy rib locking plate rib fracture internal fixation}

During the operation, the center position of multiple rib fractures was selected according to the location and shape of the fracture (the approximate center point of the maximum lateral and longitudinal line of the multiple lateral rib fracture area) (Fig. 2).

For the multiple rib fractures away from the center of the incision, the tunnel-type rib fracture internal fixation system is used for fixation. If the fracture is obviously dislocated, the periosteum is cut along the fracture line and the reduction clamp is used to pull the reduction. The internal fixation material is selected from the Synthes MatrixRIB titanium alloy rib locking plate fixing system (Johnson \& Johnson). The rib cage is selected according to the shape of the rib. The rib bone plate is drilled and fixed with 5-6 pieces of locking pin with a length of $8 \mathrm{~mm}$ and a diameter of $3 \mathrm{~mm}$, and the fracture end is carefully avoided. The procedure has the advantages of small trauma, simple operation, safety, liable fixation, good tissue compatibility and less complications. It is suitable for rib fractures in special parts of costal cartilage and paraspinal, especially ideal for patients with complications, such as floating thoracic wall, flail chest and blood pneumothorax.

\section{Video-assisted thoracoscopic assisted minimally inva- sive rib fracture open reduction and internal fixation}

Video-assisted thoracoscopic techniques have been applied to thoracic exploration and hemostasis in the treatment of chest trauma, which have become necessary technical means for the treatment of chest trauma $(11,12)$.

Video-assisted thoracoscopic surgery can observe the place where the chest wall floating is more serious, and guide the reasonable choice of surgical incision. During the operation, the thoracic cavity, lung and diaphragm muscle injury can be detected and treated in time. Thoracoscopy is used to remove the functionalized hemothorax to maximize the space for lung recruitment. However, there are few reports on the application of open reduction and internal fixation for rib fractures. The reason is that the bony thorax is tightly combined 
Table I. Detailed partition of fractures.

\begin{tabular}{|c|c|c|c|c|c|}
\hline Partition & Position & Rib range & Incision position & Muscle involved & Nerve involved \\
\hline 1 & Supine & 2-6 front ribs & $\begin{array}{l}\text { Curved incision of the medial } \\
\text { edge of the pectoralis major }\end{array}$ & Pectoralis major & \\
\hline 2 & $\begin{array}{l}\text { Supine } \\
\text { /lateral }\end{array}$ & $7-10$ front ribs & Rib bow incision & $\begin{array}{l}\text { Extra-abdominal } \\
\text { oblique }\end{array}$ & \\
\hline \multirow[t]{3}{*}{3} & Lateral & 2-6 side front ribs & $\begin{array}{l}\text { Lateral marginal incision of } \\
\text { the pectoralis major muscle } \\
\text { (anterior axillary line incision) }\end{array}$ & Pectoralis major & Long thoracic nerve \\
\hline & & $3-10$ side ribs & Midaxillary line incision & Front serratus & Thoracic dorsal nerve \\
\hline & & 3-10 side rear ribs & Posterior axillary line incision & Latissimus dorsi & Thoracic dorsal nerve \\
\hline 4 & Prone & $2-5$ rear ribs & Scapular medial edge incision & Trapezius rhomboid & \\
\hline 5 & Prone & 5-8 rear ribs & Auscultation triangle incision & $\begin{array}{l}\text { Trapezius, latissimus } \\
\text { dorsi }\end{array}$ & \\
\hline 6 & Prone & 5-12 paravertebral ribs & $\begin{array}{l}\text { Paravertebral longitudinal } \\
\text { incision }\end{array}$ & $\begin{array}{l}\text { Trapezius, latissimus } \\
\text { dorsi, erector spine }\end{array}$ & \\
\hline 7 & $\begin{array}{l}\text { Prone } \\
\text { /lateral }\end{array}$ & $7-12$ rear ribs & $\begin{array}{l}\text { Posterolateral oblique incision } \\
\text { (inclined incision along } \\
\text { the latissimus dorsi fibers) }\end{array}$ & $\begin{array}{l}\text { Latissimus dorsi, } \\
\text { serratus anterior and } \\
\text { posterior }\end{array}$ & \\
\hline
\end{tabular}

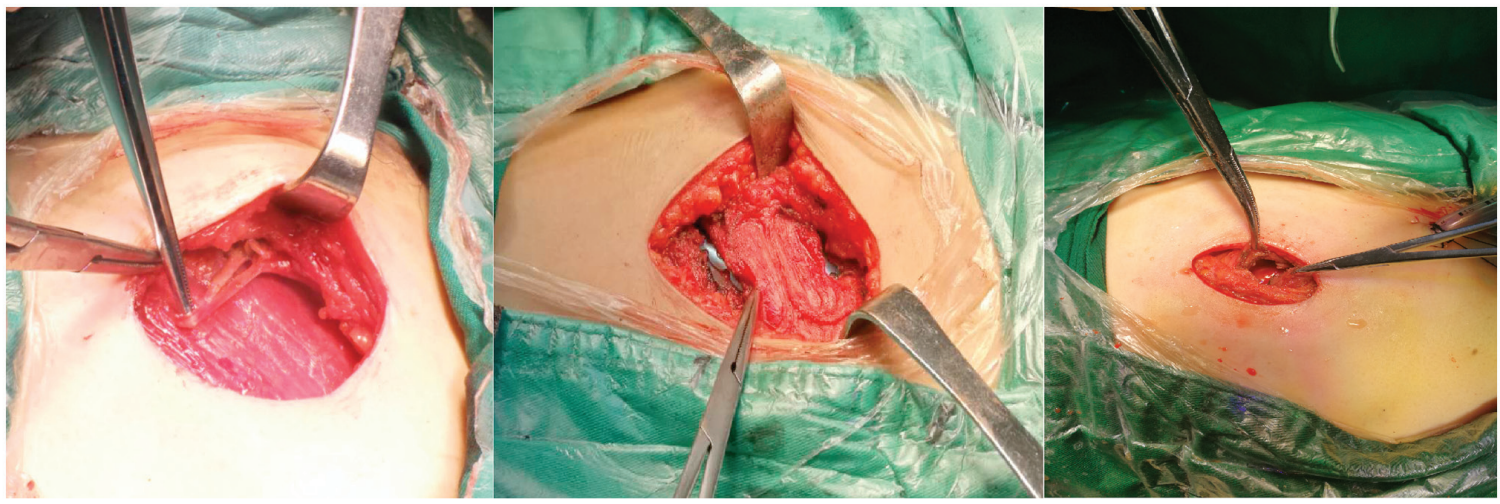

Figure 1. Small incision surgery.

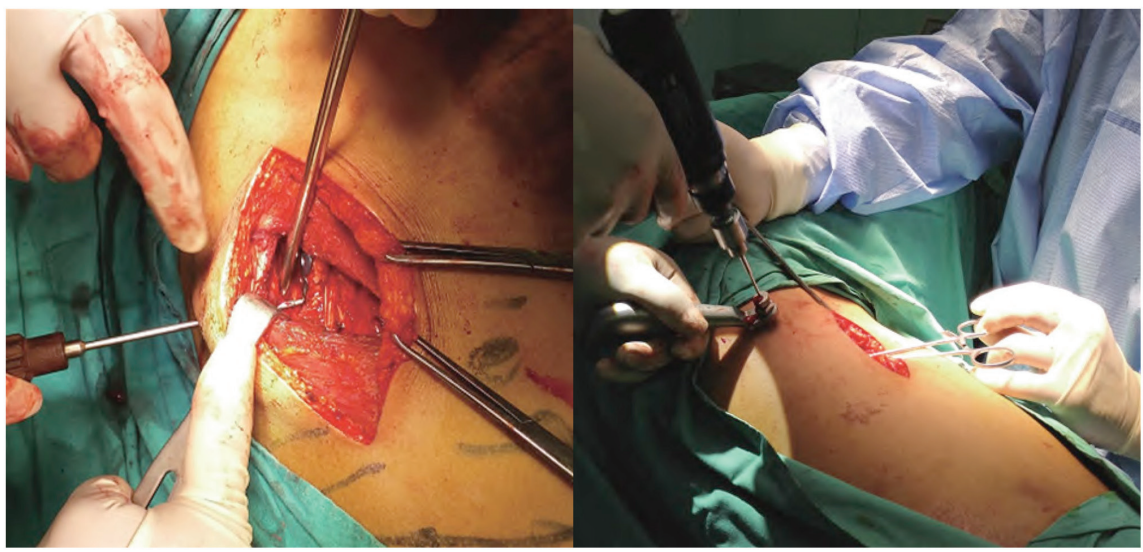

Figure 2. Rib fracture internal fixation with Synthes MatrixRIB titanium alloy rib locking plate.

with the soft tissue of the chest wall. There is no conventional internal distraction surgical instrument to provide operation space for surgery and few surgical instruments are suitable for minimally invasive rib fracture internal fixation. 
Tunnel-type thoracoscopic rib fracture fixation. Positive results of the application of endoscopic techniques have been reported. The author team developed and designed a surgical device that can be used to open the muscular chest wall from inside, and the rib plate clamp for the endoscope, the periosteal free device, and the endoscope bracket (Fig. 3). The chest wall expander can open the thoracic and muscular thorax, providing an operating space, making it possible to apply the video-assisted thoracoscopic technique to the open reduction and internal fixation of the rib fracture. The endoscopic periosteal free device can absorb a large amount of smoke during most of the operation to ensure the clarity of the surgical field of view. The rib plate clamp for the laparoscopic plate can push the rib fixation plate along the rib direction and buckle on both ends of the anatomical reduction rib fracture. At the same time, the special rib clamp is used to fix the rib fixing plate to the fracture end surface. Due to the small operation space, the existing clamp can flexibly adjust the angle, which is suitable for minimally invasive surgery. The skeletal thoracic tunnel endoscopic rib internal fixation operation is realized, which can effectively reduce the length of the incision, reduce the muscle damage of the chest wall, fully reveal the surgical field, reduce the surgical trauma, and save the operation time and manpower. Thus, this technique has obvious advantages compared with conventional incision surgery (13).

The specific surgical procedure is as follows: General anesthesia by single lumen cannula, the patient's surgical position is designed according to the rib fracture site and the number of fractures (supine position, prone position, or left and right lateral position), and if necessary, the position can be changed during surgery; the $3 \mathrm{D}$ reconstruction data of the ribs, the surface positioning measuring scale and the CT surface positioning technique of the CT fractures are combined to mark the surface location of the fracture end. The surgical incision is located in combination with the anatomical distribution of the chest wall muscles of the fracture site, generally taking into consideration the central position of the multiple rib fracture and the chest wall muscle space (commonly used approaches: Pectoralis major approach, breast inferior margin approach, auscultation triangle approach, erector spine approach and axillary approach).

The incision can be transverse, longitudinal or oblique, $\sim 3-5 \mathrm{~cm}$ long, free subcutaneous and muscular layer, dissected along the muscle space and muscle texture to the skeletal thoracic surface, avoiding breaking muscles; to cut part of the muscle attachment point along the bony thoracic surface; a special chest wall opener is used to open the surgical instrument to prop up the muscular thorax in a direction perpendicular to the skeletal thorax (the chest wall opener has completed the application of the National utility model patent, application no. 201721524911.X). To separate the skeletal thoracic and muscular thoracic the thoracoscope is inserted, the necessary muscular thorax is freed under the guidance of the endoscope, and form a temporary chest wall tunnel. After reduction of the fracture of the distal incision, the rib fixation plate should be buckled at the ends of the rib fractures on the anatomical reduction along the direction of the ribs, and the rib fixation plates were circum ferentially fixed to the fracture end surface using special clamps. The internal fixation material can be determined according to the fracture site of the patient and the economic conditions of the patient. For example, the rib cage locking plate can be combined with the rib minimally invasive plate system (MatrixRIB-MIPO; Johnson \& Johnson). Compared with conventional surgical video-assisted thoracoscopic surgery, this procedure can provide good illumination, visually and comprehensively explore the tissue structure around the fracture end, determine the specific location, number and severity of the rib fracture, and effectively avoid damage to the chest wall muscle and intercostal vessel and nerve, significantly reducing postoperative complications. Therefore, it is worthy of clinical promotion.

\section{The Su total thoracoscopic rib fracture bone plate nail intrathoracic implantation and fixation}

Thoracoscopic implanted NiTi memory alloy rib plate intrathoracic implantation and thoracoscopic absorbable rib nail fixation were performed.

The indications for laparoscopic surgery were as follows: i) Patients with fractures in the F-zone, i.e., non-occlusion of the axillary, anterior and posterior and lateral chest walls; ii) single or multiple fractures or simultaneous laparoscopic repair of vascular injuries. Surgical fractures should be in the non-occluded area of the axillary, anterior and posterior and lateral chest walls. The Su total thoracoscopic rib fractures and bone plate fixation in the thoracic cavity are technically feasible in selected patients, however, they are not replaceable in severe cases, such as conventional thoracotomy for open thoracic surgery, which requires further improvement. Pieracci et al (14) reported the use of locking plate fixation in the thoracic cavity to fix the ribs in the thoracic cavity. The advantage is that the thoracic cavity can be explored while fixing the ribs, however, the operation in the thoracic cavity is limited, the fracture reduction is difficult, and has high requirements.

\section{Minimally invasive Nuss surgery for patients with severe flail chest with sternal fracture}

Lee and Kang (15) reported the use of Nuss method for the treatment of bilateral vertebral fractures with sternal fractures and sacral thoracotomy (Fig. 4); combination of sternal fractures and severe flail chest is a clinically life-threatening disease, and the conventional sternal approach uses a metal plate to stabilize the chest wall, which does not achieve the effect of stabilizing the chest wall. Nuss surgery is used to support the collapse of the chest wall from the posterior sternum. The chest wall is stabilized after surgery. The operation uses the midline incision on both sides of the chest for $\sim 3 \mathrm{~cm}$. A subcutaneous tunnel is established between the 4 th intercostal space. The Kelly forceps is placed under the sternum and above the mediastinum. The Kelly forceps is placed from one side of the chest wall incision to the opposite side, and then $36-\mathrm{cm}$ pectus rod is pre-plasticized to form a symmetrical arch, pointing to the opposite side along the subcutaneous tunnel, rotating the Pectus rod, and then the collapsed thorax is propped up.

This operation is suitable for patients with severe flail chest combined with thoracic fracture with short operative time and small surgical injury. It has certain advantages in thoracic trauma rescue surgery, however, it has poor effect on local rib fracture reduction. The risk for postoperative delayed 


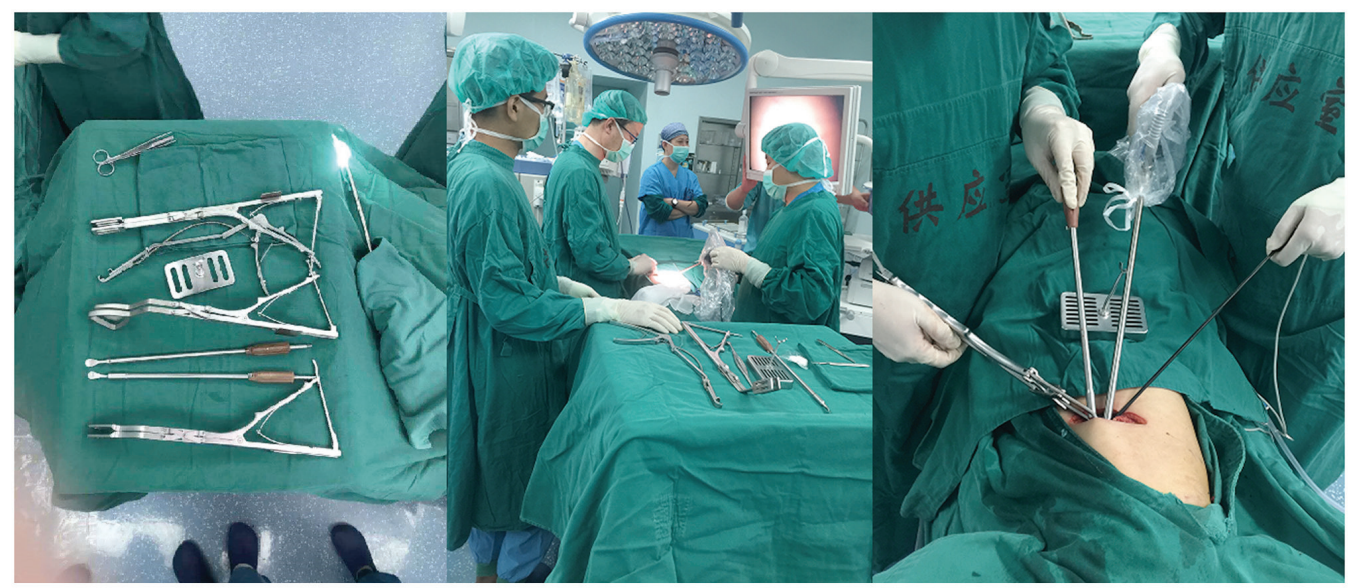

Figure 3. Patented surgical instrument for tunneled endoscopic minimally invasive rib fracture internal fixation surgery.

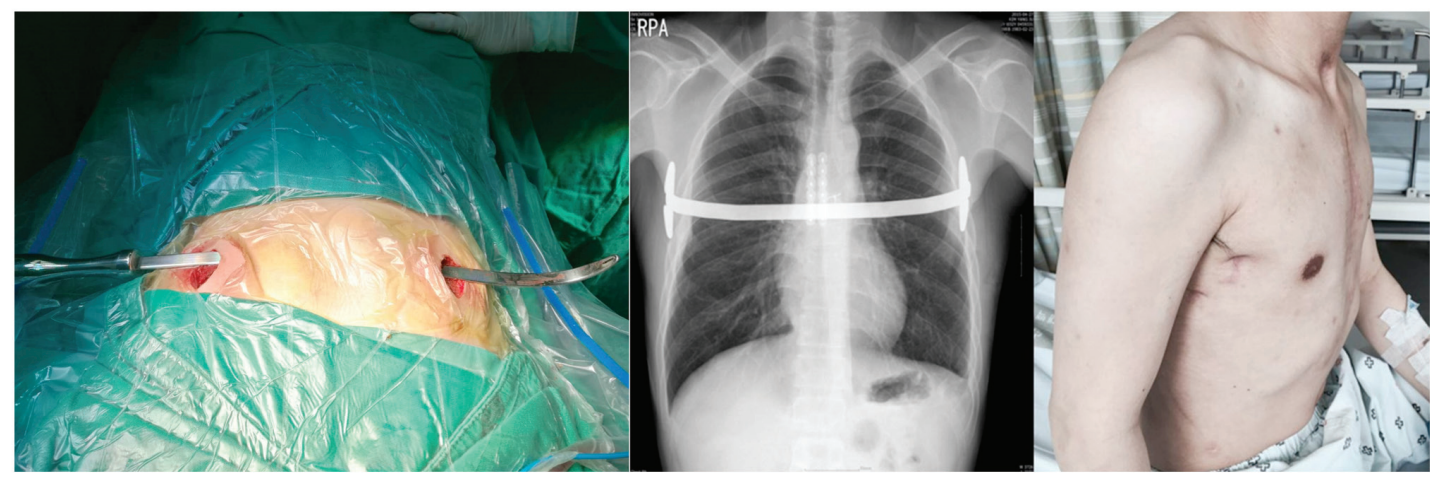

Figure 4. Nuss surgery for the treatment of flail chest.

thoracic hemorrhage is high. Unless the patient feels unwell after surgery, the orthopedic plate left in the body will not be removed. If the patient has obvious chest discomfort or demands, the orthopedic plate will be removed 1 year after the thorax is stabilized.

\section{Introduction of internal fixation materials suitable for different surgical methods}

At present, the commonly used fixing materials in China include intramedullary fixation, embracing fixation and steel plate screw fixation, which are respectively represented by absorbable rib nails, nickel-titanium or memory alloy plates, and MatrixRIB titanium plates, each with advantages and disadvantages $(16,17)$. Among them, the nickel-titanium alloy embracing fixation is a simple operation procedure, which does not require excessive peeling of the periosteum during surgery, and is suitable for patients with a large number of fractures.

Because the embracing device easily compresses the intercostal nerve, there is a possibility that the pain cannot be relieved after surgery, and fractures near the rib cartilage and the spine are not suitable for application; pure titanium alloy embracing device requires fully peeling the periosteum to accurately reset the fracture end, and the fixation effect is exact, although the operation is complicated. As a result, it is not suitable for rib fractures far from the incision, or for rib fractures near costal cartilage and the spine. Titanium alloy rib locking plate for internal fixation of rib fractures has a good therapeutic effect for some cases that are difficult to reset or with multiple fractures (18). Small incision or tunnel laparoscopic surgery can be combined with the rib minimally invasive plate system (MatrixRIB-MIPO), which can accurately shape and firmly fix rib fractures in multiple locations, especially for fractures near costal cartilage and the spine (19). The absorbable rib nail or plate is made of polylactic acid polymer, which has been successfully applied to traumatic flail chest and non-traumatic thoracotomy (20). It has not been widely clinically popularized because of its limitations in biomechanics and degradation rate.

\section{Conclusion}

At present, surgical treatment of multiple rib fractures has been accepted by an increasing number of medical workers (21-23), and surgery tends to be minimally invasive. Different rib fixing materials have their own advantages and disadvantages. Nickel-titanium alloy embracing fixator is a new material with memory function at temperature. It can be stretched and deformed at low temperature, automatically restored to its original state under body temperature, without secondary surgery. It has good histocompatibility, strong corrosion resistance, less immune rejection, and does not affect magnetic resonance imaging. The pure titanium claw-shaped bone plate has high strength, good histocompatibility, and no need for 
secondary surgery. The advantages are similar to those of the nickel-titanium alloy embracing fixator, however, special tools are required for fixing and removing, and a large space is required for operation. The absorbable intramedullary nail has a slightly higher flexural strength than the human cortical bone, and is completely degraded in 3-5 years. The effective support time is $8-10$ months. It does not require secondary surgery, and the fracture healing can be stimulated during the decomposition process. However, there are certain deficiencies in clinical application. The stability of the internal fixation is slightly worse than that of the nickel-titanium alloy embracing fixator and the pure titanium claw-shaped bone plate. For the fractured wedge or comminuted fracture, it is usually not fixed. The development and clinical application of absorbable rib fixation materials will be a research hotspot in the field of chest trauma treatment. The clinical promotion of absorbable materials in multiple rib fracture surgery will make the surgery even less invasive, reducing surgical complications $(24,25)$.

3D printing technology has been widely used in various medical departments, such as the production of personalized artificial prostheses and built-in objects $(26,27)$; especially for some complex rib fractures, 3D printing technology is adopted. According to the rib fracture reconstruction model one day before surgery, the corresponding rib internal fixation materials and models, and individualized plasticity are selected to complete accurate preoperative planning; thereby reducing or even eliminating the internal fixation shaping time in tunnel surgery, further reducing the surgical injury of the patient. The development of surgical instruments specially used to open the musculoskeletal thoracic space provides an operating space for laparoscopic surgery, opening up a new range of clinical use of thoracoscopic surgery.

In conclusion, with in-depth understanding of the minimally invasive concept, the accumulation of clinical experience and the unremitting exploration of technology, the continuous improvement of fixed materials and surgical instruments for minimally invasive surgery with less trauma and less operation time will be used clinically to achieve a satisfactory clinical outcome.

\section{Acknowledgements}

Not applicable.

\section{Funding}

No funding was received.

\section{Availability of data and materials}

Not applicable.

\section{Authors' contributions}

HX, DZ, JL, ZS and DW were involved in the conception and design of this study. LD, PZ, YZ and XL were responsible for the literature research and drafted the manuscript. DZ and DW made revisions from critical perspective for important intellectual content. The final version was read and approved by all authors.

\section{Ethics approval and consent to participate}

Not applicable.

\section{Patient consent for publication}

Not applicable.

\section{Competing interests}

The authors declare that they have no competing interests.

\section{References}

1. Ho XN, Wee IJ, Syn N, Harrison M, Wilson L and Choong AM: The endovascular repair of blunt traumatic thoracic aortic injury in Asia: A systematic review and meta-analysis. Vascular 27: 213-223, 2019.

2. Brasel KJ, Moore EE, Albrecht RA, deMoya M, Schreiber M, Karmy-Jones R, Rowell S, Namias N, Cohen M, Shatz DV, et al: Western trauma association critical decisions in trauma: Management of rib fractures. J Trauma Acute Care Surg 82: 200-203, 2017.

3. Pieracci FM, Majercik S, Ali-Osman F, Ang D, Doben A, Edwards JG, French B, Gasparri M, Marasco S, Minshall C, et al: Consensus statement: Surgical stabilization of rib fractures rib fracture colloquium clinical practice guidelines. Injury 48: 307-321, 2017.

4. Bemelman M, de Kruijf MW, van Baal M and Leenen L: Rib fractures: To fix or not to fix? An evidence-based algorithm. Korean J Thorac Cardiovasc Surg 50: 229-234, 2017.

5. Kocher GJ, Sharafi S, Azenha LF and Schmid RA: Chest wall stabilization in ventilator-dependent traumatic flail chest patients: Who benefits? Eur J Cardiothorac Surg 51: 696-701, 2017.

6. Farquhar J, Almarhabi Y, Slobogean G, Slobogean B, Garraway N, Simons RK and Hameed SM: No benefit to surgical fixation of flail chest injuries compared with modern comprehensive management: Results of a retrospective cohort study. Can J Surg 59: 299-303, 2016.

7. Uchida K, Nishimura T, Takesada H, Morioka T, Hagawa N, Yamamoto T, Kaga S, Terada T, Shinyama N, Yamamoto H, et al: Evaluation of efficacy and indications of surgical fixation for multiple rib fractures: A propensity-score matched analysis. Eur J Trauma Emerg Surg 43: 541-547, 2017.

8. Pieracci FM, Lin Y, Rodil M, Synder M, Herbert B, Tran DK, Stoval RT, Johnson JL, Biffl WL, Barnett CC, et al: A prospective, controlled clinical evaluation of surgical stabilization of severe rib fractures. J Trauma Acute Care Surg 80: 187-194, 2016.

9. de Campos JRM and White TW: Chest wall stabilization in trauma patients: Why, when, and how? J Thorac Dis 10 (Suppl 8): S951-S962, 2018.

10. Taylor BC, French BG and Fowler TT: Surgical approaches for rib fracture fixation. J Orthop Trauma 27: e168-e173, 2013.

11. Berninger MT, Kellermann F, Woltmann A, Bühren V and Lang M: Single-port VATS-assisted internal fixation of serial rib fractures. Unfallchirurg 121: 335-338, 2018 (In German).

12. Fraser SF, Tan C, Kuppusamy MK, Gukop P and Hunt IJ: The role of a video-assisted thoracic approach for rib fixation. Eur J Trauma Emerg Surg 43: 185-190, 2017.

13. Xia H, Zhu P, Li J, Zhu D, Sun Z, Deng L, Zhang Y and Wang D: Thoracoscope combined with internal support system of chest wall in open reduction and internal fixation for multiple rib fractures. Exp Ther Med 16: 4650-4654, 2018.

14. Pieracci FM, Johnson JL, Stovall RT and Jurkovich GJ: Completely thoracoscopic, intra-pleural reduction and fixation of severe rib fractures. Trauma Case Rep 1: 39-43, 2015.

15. Lee SK and Kang K: Nuss procedure for surgical stabilization of flail chest with horizontal sternal body fracture and multiple bilateral rib fractures. J Thorac Dis 8: E390-E392, 2016.

16. Klein GT, Lu Y and Wang MY: 3D printing and neurosurgery ready for prime time? World Neurosurg 80: 233-235, 2013.

17. Nolasco-de IRAL, Mosiñoz-Montes R, Matehuala-García J, Román-Guzmán E, Quero-Sandoval F, Reyes-Miranda AL: Unstable thorax fixation with bioabsorbable plates and screws. Presentation of some cases. Cir Cir 83: 23-28, 2015. 
18. Benian AS, Pushkin SIu, Syzrantsev IuV and Kameev IR: Osteosyntesis of ribs using the technology 'matrix rib' in treatment of victims with multiple float rib fractures. Vestn Khir Im I I Grek 172: 78-79, 2013 (In Russian).

19. Vana PG, Neubauer DC and Luchette FA: Contemporary management of flail chest. Am Surg 80: 527-535, 2014.

20. Gao E, Li Y, Zhao T, Guo X, He W, Wu W, Zhao Y and Yang Y: Simultaneous surgical treatment of sternum and costal cartilage fractures. Ann Thorac Surg 107: e119-e120, 2019.

21. Galos D, Taylor B and McLaurin T: Operative fixation of Rib fractures indications, techniques, and outcomes. Bull Hosp Jt Dis (2013) 75: 15-20, 2017.

22. Tarng YW, Liu YY, Huang FD, Lin HL, Wu TC and Chou YP: The surgical stabilization of multiple rib fractures using titanium elastic nail in blunt chest trauma with acute respiratory failure. Surg Endosc 30: 388-395, 2016.

23. Dehghan N: Challenges in plate fixation of chest wall injuries. Injury 49 (Suppl 1): S39-S43, 2018.
24. Morodomi Y, Okamoto T, Tagawa T, Shoji F, Katsura M, Fujishita T, Fujiyoshi T, Akahoshi T, Yasuda M and Maehara Y: A novel method of using bioabsorbable materials for the surgical repair of flail chest. J Trauma Acute Care Surg 81: 984-987, 2016.

25. Nolasco-de la Rosa AL, Mosiñoz-Montes R, Matehuala-García J, Cuautle-Ramírez AA, Román-Guzmán E, Reyes-Miranda AL and Quero-Sandoval F: Thoracic inestability fixed with bioabsorbable screws and plates. Acta Ortop Mex 30: 311-315, 2016 (In Spanish).

26. Kim YH, Park Y and Chung KJ: Considerations for the management of medial orbital wall blowout fracture. Arch Plast Surg 43: 229-236, 2016.

27. Leinicke JA, Elmore L, Freeman BD and Colditz GA: Operative management of rib fractures in the setting of flail chest: A systematic review and meta-analysis. Ann Surg 258: 914-921, 2013.

This work is licensed under a Creative Commons

Attribution-NonCommercial-NoDerivatives 4.0 International (CC BY-NC-ND 4.0) License. 